\title{
Lapurdum
}

Euskal ikerketen aldizkaria | Revue d'études basques |

Revista de estudios vascos | Basque studies review

19 | 2016

Numéro XIX

\section{Gestion des cas absolutif et ergatif en basque par des bilingues précoces basques-français}

\author{
Isabelle Duguine
}

\section{OpenEdition}

Journals

Édition électronique

URL : https://journals.openedition.org/lapurdum/3323

DOI : 10.4000/lapurdum.3323

ISSN : 1965-0655

Éditeur

IKER

Édition imprimée

Date de publication : 1 janvier 2016

Pagination : 201-217

ISBN : 978-2-95534-134-6

ISSN : $1273-3830$

Référence électronique

Isabelle Duguine, «Gestion des cas absolutif et ergatif en basque par des bilingues précoces basquesfrançais », Lapurdum [En ligne], 19 | 2016, mis en ligne le 01 janvier 2021, consulté le 03 septembre 2021. URL : http://journals.openedition.org/lapurdum/3323 ; DOI : https://doi.org/10.4000/lapurdum. 3323

Creative Commons - Attribution - Pas d'Utilisation Commerciale - Pas de Modification 4.0 International - CC BY-NC-ND 4.0 


\section{Gestion des cas absolutif et ergatif en basque par des bilingues précoces basques-français}

Isabelle DUGUINE

Université de Pau et des Pays de l'Adour - IKER UMR 5478

\section{Résumé}

Dans cet article, nous observons la gestion des morphèmes marqués et non marqués illustrés par les cas ergatif et absolutif en basque chez des enfants bilingues basques-français et monolingues bascophones âgés en moyenne de cinq ans. Pour ce faire, nous avons sélectionné neuf enfants parlant le basque, monolingues et bilingues de profils différents. Nous leur avons soumis deux épreuves en langage contrôlé pour la production des deux marqueurs morphologiques ainsi qu'une épreuve de compréhension. Les résultats montrent des différences significatives dans la production du cas ergatif (morphème marqué) par rapport au cas absolutif (morphème non marqué). Emprunté au champ de la pathologie du langage, une des hypothèses explicatives avancées dans le cadre de cet article est la complexité structurale du cas ergatif pouvant entraîner une charge cognitive importante et aboutissant au final à l'omission du morphème ergatif.

\section{Introduction}

Cet article se veut une contribution à l'étude de la langue basque, encore peu étudiée dans le domaine de l'acquisition. Ainsi, l'objectif principal est de vérifier l'hypothèse selon laquelle le morphème zéro serait plus facile à gérer que le morphème marqué en l'appliquant à la morphologie nominale basque, celle-ci comprenant pas moins d'une dizaine de cas de déclinaisons. Nous nous intéressons uniquement à deux cas : l'absolutif (un morphème non marqué) et l'ergatif (un morphème marqué), tous deux portant sur le syntagme nominal sujet. Le premier marque le sujet d'un verbe intransitif ou l'objet dans une phrase transitive tandis que le second désigne le sujet du verbe transitif (Allières, 1977 ; 1979 ; Oyharçabal, 2003 ; 2011). Avant d'exposer en détail la méthodologie suivie dans cette étude, nous allons revenir sur le cadre théorique à l'origine de l'hypothèse de la marque. Nous aborderons également la question de la charge cognitive en lien avec la complexité structurale et des stratégies de recours en cas de difficultés langagières rencontrées par le locuteur. 


\section{Cadre théorique}

\subsection{Retour sur la théorie de la marque}

La théorie de la marque est née dans les années 1930 à la suite d'échanges épistolaires entre Troubetskoy et Jakobson, tous deux membres du Cercle de Prague et appartenant au mouvement de réflexion structuraliste. Elaborée au départ pour expliquer l'opposition entre les phonèmes marqués et les phonèmes non-marqués, la théorie de la marque a ensuite été étendue à d'autres domaines linguistiques comme la morphologie ou la sémantique (Jakobson et Waugh, 1980 ; Gadet, 1994). Cette notion importante pour l'analyse des systèmes linguistiques postule l'existence d'une opposition entre éléments marqués -qui seraient nécessairement « positifs » et « actifs »- et non marqués- à l'inverse, « négatifs » et « passifs». Sur le plan phonologique, les exemples sont nombreux. Jakobson et Waugh (1980 : 113) mentionnent la dyade entre « consonne palatalisée et consonne non palatalisée » ou encore celle entre « consonne labialisée et consonne non labialisée ». Cette dichotomie entre formes marquées et non marquées se conçoit de manière hiérarchique selon Jakobson (1969). Il dit ceci : «l'idée de marque devient particulièrement indispensable dès qu'il s'agit d'expliquer une superposition quelconque de valeurs linguistiques et d'interpréter la structuration hiérarchique de la langue humaine et l'ordre de la construction du langage » (Jakobson, 1969 : 9). Par exemple, elle sert à comprendre l'ordre d'acquisition du système phonologique chez l'enfant qui débuterait par les structures les moins marquées pour aller vers les structures les plus marquées, soit du plus simple au plus complexe. Ainsi, l'acquisition des dentales précèderait toujours celle des palatales dans le langage enfantin (Jakobson, 1969 : 51) et ce, quelle que soit la langue. Il semblerait également que les consonnes sourdes /p t k/ soient acquises plus tôt que les consonnes sonores /b d g/ (Dordain et al. 1992). En somme, les unités linguistiques c'est-à-dire les phonèmes et les morphèmes, ne se situent pas sur un même pied d'égalité : elles seraient hiérarchiquement organisées en fonction de leur complexité structurale intrinsèque. Or, si la hiérarchisation des formes linguistiques peut s'exprimer en termes de complexité structurale, cela pourrait expliquer en partie les erreurs de performance observées chez l'enfant comme chez l'adulte. Certaines voies de recherche notamment dans le domaine aphasiologique semblent aller dans ce sens comme nous allons le voir ci-après.

\subsection{Hiérarchie des unités linguistiques et complexité structurale}

Les données issues des études sur la pathologie du langage suggèrent que la gestion des phonèmes non-marqués -structuralement moins complexes- serait plus aisée que celle des phonèmes marqués -structuralement plus complexes- (Nespoulous, 2004). Pour preuve, face à la complexité structurale contenue dans certains groupes consonantiques de la langue française comme /bR/ /pR//dR/ etc., le patient aphasique aurait tendance à « simplifier la structure syllabique en remplaçant les structures branchantes de type Consonne-ConsonneVoyelle (CCV) par des structures plus simples de type CVCV» (Nespoulous, 2004 : 183). Le mot/bRwet/ deviendrait selon la stratégie utilisée /bwet/ par syncope : c'est-à-dire que le patient élide la consonne liquide /R/ ou bien /beRwet/ par épenthèse : ici, il insère une voyelle entre les deux consonnes (Nespoulous, 1996 ; Nespoulous et Virbel, 2004). En clair, le groupe consonantique $/ \mathrm{bR} /$ serait plus difficile à produire que la consonne sonore $/ \mathrm{b} /$ seule. 
En outre, selon Nespoulous et Moreau (1998), la syncope consonantique atteindrait toujours un certain type de consonnes : soit la liquide placée en deuxième position dans un groupe formé par la paire /occlusive + liquide/ (ex. : /pR/) ou par la suivante /fricative + liquide/ (ex. : /fR/) soit la fricative à l'initiale dans le groupe /fricative + occlusive/ comme /sp/, les occlusives étant préservées dans les deux cas. De manière analogue, mais cette fois au niveau morphologique, les morphèmes marqués seraient plus difficiles à gérer que les morphèmes non-marqués (Dordain et Nespoulous, 1992). Ces mêmes chercheurs prennent l'exemple de la conjugaison du verbe « parler » qui donne/parlœre/ au futur, c'est-à-dire une structure plus complexe que celle du présent /parl/ en raison du nombre de marques à gérer. En résumé, les contraintes linguistiques (ici morphologiques) se manifestent par des difficultés langagières qui conduisent généralement à une simplification de la structure cognitivement coûteuse.

\subsection{Hypothèse du lien entre la complexité structurale et le poids cognitif}

L'hypothèse d'une corrélation entre la complexité structurale et la charge procédurale a été suggérée par Dordain et Nespoulous (1988), l'idée étant que plus le nombre d'opérations mentales est important, plus la charge cognitive augmente et plus le sujet est susceptible de faire des erreurs (Dordain et Nespoulous, 1992). Rappelons que la charge cognitive mesure la quantité de ressources mentales mobilisées par un sujet lors de la réalisation d'une tâche et des capacités propres à l'individu (Chanquoy et al., 2007 : 248). Selon ces auteurs, l'intensité du traitement cognitif sollicité pour la réalisation d'une tâche peut être précisée si l'on tient compte des paramètres comme le nombre d'informations à stocker en mémoire de travail, les pré-requis de l'individu quant à la tâche, ses stratégies personnelles pour trier l'information, sa motivation ou encore son état de fatigue.

La limitation des ressources attentionnelles et/ou mnésiques est une explication avancée dans le domaine aphasiologique pour justifier les erreurs commises en production (Nespoulous, 2006). Les capacités cognitives chez le sujet aphasique étant limitées, il est obligé de faire des choix lors des traitements des messages linguistiques et c'est le plus souvent la morphologie qui en pâtit au profit de "niveaux de traitement plus élevés parce que plus cruciaux compte tenu de l'enjeu sémiotique de l'acte de communication ou de la tâche complexe en cours» (Nespoulous, 2006 : 5). La gestion de calculs plus profonds (de type syntaxique) serait plus difficile que celle des calculs superficiels (de type morphologique). Lors du traitement du message linguistique, la morphologie, plus superficielle, serait donc négligée par rapport à la syntaxe qui représenterait un niveau de traitement plus élevé. De la même manière, d'après Delage (2008), de nombreux travaux montrent que les enfants comportant des troubles spécifiques du langage produiraient davantage d'erreurs sur les éléments requérant un calcul syntaxique plus complexe. Par ailleurs, le degré de complexité influence l'acquisition de la syntaxe chez l'enfant dans le sens où les énoncés les plus complexes sont maîtrisés plus tardivement (Delage et al., 2012). Ce propos vient soutenir l'hypothèse de Jakobson précédemment énoncée (1969) et reprise plus récemment par Kail (1997) selon laquelle le degré de la complexité structurale aurait un impact sur la chronologie des acquisitions langagières en ce sens que les structures simples seraient acquises avant les structures complexes. 


\subsection{Difficultés langagières et stratégies palliatives}

A la manière de Jakobson (1969) qui établit un lien entre le langage de l'aphasique et celui de l'enfant, les erreurs observables chez l'enfant sans troubles seraient pour une grande part dues à une capacité de traitement linguistique limitée. Ce déficit est probablement à mettre en relation avec l'état immature de son cerveau et/ou de la quantité réduite de son expérience linguistique (Kolk, 2001). C'est aussi une des raisons invoquées par Duvignau et al. (2004) pour expliquer le vocabulaire déficitaire de l'enfant à un âge très précoce (autour de deux ans). Ces auteurs ajoutent qu'à l'instar de l'aphasique, l'enfant fera appel à des stratégies de sur-extension catégorielles (ex. : 'pomme' pour orange) ou analogiques (ex. : 'chapeau' pour un capuchon de stylo) sémantiquement proches du mot-cible pour pallier ce manque du mot. Nous n'irons pas plus loin dans l'examen des stratégies linguistiques car il y a en a beaucoup d'autres : des stratégies périphrastiques aux stratégies non verbales de type gestuel -en particulier dans les situations de manque du mot- en passant par les stratégies de réduction quantitative, d'auto-correction et d'évitement (Nespoulous, 1996 ; Sahraoui et Nespoulous, 2008 ; Sahraoui, 2009). Lidée principale à retenir à travers ces travaux en aphasiologie est l'aptitude de l'individu à pouvoir s'adapter lorsqu'il se retrouve en situation difficile et que ses capacités cognitives et linguistiques ne lui permettent pas d'y faire face. Il va alors utiliser des outils alternatifs qu'il a en sa possession dans le but de communiquer et de se faire comprendre.

Pour en revenir à la morphologie nominale qui nous intéresse ici, la question est de savoir si la gestion mentale d'un morphème marqué est plus difficile que celle d'un morphème non marqué (ou zéro). On peut se demander si un calcul qui conduit à un morphème zéro a vraiment le même poids cognitif qu'un calcul qui conduit à un morphème exprimé. En raison de sa morphologie agglutinante, la langue basque se prête adéquatement comme objet d'étude pour tenter de répondre à cette question.

\subsection{Morphologie du basque et acquisition}

Les lignes suivantes présentent quelques caractéristiques générales de la langue basque et sont destinées au lecteur non averti.

\subsubsection{Le basque : une langue ergative}

D’origine pré-indo-européenne, la langue basque possède une morphologie nominale agglutinante. Parmi la dizaine de cas de déclinaison, le cas ergatif, représenté par le morphème -k, désigne le sujet du verbe transitif (cf. ci-après (1)) alors que le cas absolutif (morphème zéro) marque le sujet d'un verbe intransitif ou l'objet dans une phrase transitive (cf. 2) (Allières, 1977 ; 1979 ; Oyharçabal, 2003 ; 2011).

Gizona- $\Theta$

homme-art.sg.ABS

'Lhomme est venu'

(2) Gizona-k

homme-art.sg.ERG

'Lhomme a vu le chat' jin da

venir.perf aux.être

$\begin{array}{lll}\text { gatua- } \Theta & i k u s i & d u \\ \text { chat-art.sg.abs } & \text { voir.perf } & \text { aux.avoir }\end{array}$


D'autre part, l'ergativité suppose un double marquage affectant à la fois la morphologie nominale et la morphologie verbale par la présence d'indices au niveau de l'auxiliaire ${ }^{1}$. Le verbe s'accorde avec tous ses actants (sujet et objets). Ainsi, la structure morphologique du verbe peut contenir jusqu'à trois marques en relation avec les cas grammaticaux (absolutif, ergatif, datif). Autrement dit, le verbe basque comprend les indices de personne renvoyant au sujet du verbe intransitif ou à l'objet direct pour les verbes transitifs (d'où le lien avec le cas absolutif), au sujet de l'action (ici l'ergatif) et au bénéficiaire tant pour les transitifs que pour les intransitifs (ici le datif). Notons aussi que le basque est une langue pro-drop et que, plus généralement, le marquage morphologique des actants dans le verbe peut s'accompagner d'une absence de marquage pronominal (de ces actants) dans la phrase. Enfin, l'ordre des mots en basque est relativement libre en raison des cas de déclinaison qui indiquent la fonction syntaxique de chaque constituant. Ainsi, une phrase composée d'un sujet S, d'un verbe V et d'un objet O peut admettre plusieurs ordres notamment les suivants : SOV, SVO, OSV, OVS, VSO, VOS.

\subsection{2. Âge d'acquisition des cas absolutif et ergatif}

Le cas absolutif étant un morphème zéro, il est par conséquent difficile de prouver son acquisition. En revanche, on peut toutefois se baser sur la maîtrise des articles définis singulier $-a$ et pluriel -ak au sein des syntagmes nominaux pour en déduire celle de ce marqueur. S'agissant de la production des phrases intransitives, le basque emploie l'article défini adjoint (théoriquement) du cas absolutif. A ce jour, les données provenant du questionnaire parental MacArthur CDI adapté en basque (Garcia et al., 2011) fournissent quelques indications sur l'acquisition des articles et des différents cas de déclinaison en basque. Ainsi, on notera que l'article défini singulier s'acquiert aux alentours de 12 mois et l'article pluriel vers 30 mois suggérant par là même que l'enfant bascophone maîtrise aussi la production du cas absolutif de manière précoce.

Par ailleurs, les études développementales menées en Pays Basque d'Espagne montrent que le cas ergatif pose des problèmes chez les enfants bilingues basques-espagnols et monolingues bascophones (Barreña, 1995 ; Ezeizabarrena et Larrañaga, 1996 ; Huarte, 2007). Les auteurs s'accordent sur le fait qu'il existe différentes étapes d'acquisition concernant le cas ergatif (Huarte, 2007). Les premières productions sont souvent composées de deux mots appartenant à la catégorie lexicale des adjectifs, noms, verbes ou adverbes. Elles sont dépourvues de marques morphologiques, autrement dit aucun morphème de cas ni d'aspect ni même d'auxiliaire n'est encore produit. L'émergence du cas ergatif au sein de la morphologie nominale correspond à la période où l'on observe une augmentation de la longueur des phrases et de leur complexité. Elle se situerait aux alentours de deux ans et quatre mois

1. Les exemples (1) et (2) font apparaître ici la forme verbale périphrastique composée d'une part, d'un participe porteur de la marque aspectuelle et d'autre part, d'un auxiliaire, celui-ci revêtant les indices de personne, de nombre et de temps. Le verbe basque admet également une forme synthétique réunissant à elle seule les marques de temps et de personne mais la conjugaison synthétique est moins courante et ne concerne qu'un nombre limité de verbes. 
(Barreña, 1995 ; Huarte, 2007). Toutefois, la production de ce marqueur serait irrégulière (Ezeizabarrena, 2012) et se manifesterait par des omissions selon Huarte (2007).

\subsection{Hypothèses de travail}

Dans cet article, nous posons l'hypothèse que le cas absolutif (morphème zéro) serait acquis avant le cas ergatif (morphème marqué) laissant admettre un traitement psycholinguistique plus aisé pour le premier marqueur par rapport au second. Même si les deux morphèmes ont autant de valeur linguistique, il semblerait que le premier (morphème zéro) soit plus facile à gérer que le second (morphème exprimé). Par ailleurs, selon l'hypothèse des stratégies palliatives évoquée plus haut, le locuteur en situation de difficultés langagières peut recourir à des procédés linguistiques qui lui permettent malgré tout d'assurer une communication de l'information intelligible. Ainsi, il sera également intéressant d'observer les types de stratégies mis en place par les enfants qui rencontrent des difficultés dans la maîtrise du morphème marqué, c'est-à-dire le cas ergatif.

\section{Méthodologie}

\subsection{Population}

Notre démarche se fonde sur une étude de cas multiples comptant au total neuf enfants de profils linguistiques différents. L'échantillon comprend six enfants bilingues basquesfrançais, simultanés et successifs (Ll basque ou français) et trois monolingues bascophones âgés en moyenne de six ans. S'agissant de la sélection des sujets bilingues, les critères d'inclusion tiennent compte de la situation sociolinguistique et des modes de transmission des langues basque et française en Pays Basque de France. Concrètement, la langue basque est transmise par la voie familiale et/ou par la voie scolaire avec deux systèmes possibles : le système paritaire dans lequel la moitié des enseignements se déroulent en français et l'autre moitié en basque ou le système immersif selon lequel l'enseignement a lieu majoritairement en basque avec l'introduction progressive du français vers l'âge de six-sept ans.

Les enfants bilingues de notre étude -numérotés de 1 à 6- correspondent à l'un des quatre profils suivants :

- Profil 1 : Bilingue précoce successif de L1 basque apprenant le français L2 à l'école $\left(n^{\circ} 4\right.$ et $\left.n^{\circ} 5\right)$

- Profil 2 : Bilingue précoce successif de L1 français apprenant le basque L2 à l'école $\left(n^{\circ} 6\right)$

- Profil 3 : Bilingue précoce simultané ayant appris les langues basque et française en famille et apprenant le français à l'école ( $\left.\mathbf{n}^{\circ} \mathbf{2}\right)$

- Profil 4 : Bilingue précoce simultané ayant appris les langues basque et française en famille et apprenant le basque à l'école. ( $\mathbf{n}^{\circ} \mathbf{1}$ et $\mathbf{n}^{\circ} \mathbf{3}$ )

Quant à la population contrôle, elle est composée de trois enfants monolingues basques $\left(\mathbf{n}^{\circ} \mathbf{7}, \mathbf{n}^{\circ} \mathbf{8}\right.$ et $\left.\mathbf{n}^{\circ} \mathbf{9}\right)$, autrement dit ces enfants grandissent avec le seul contact de la langue basque : cela est particulièrement vrai pour l'enfant $\mathbf{n}^{\circ} \mathbf{7}$. Celui-ci vit en milieu rural, ses parents ainsi que son entourage proche lui parlent uniquement en basque. De plus, il 
est scolarisé dans une école immersive en langue basque implanté dans son village. L'enfant n'aurait donc pas d'interactions avec des locuteurs francophones. Autrement dit, depuis sa naissance, il n'a quasiment pas été exposé à la langue française. Les deux autres monolingues bascophones possèdent également les mêmes caractéristiques s'agissant de la transmission de la langue basque. Par contre, étant donné qu'ils vivent en milieu urbain, nous pouvons supposer qu'ils sont, même de manière passive, exposés à la langue française.

\title{
3.2. Matériel
}

Le protocole de tests présenté dans cet article comprend trois épreuves : deux épreuves de production et une épreuve de compréhension que nous allons décrire ci-après.

\subsubsection{Epreuve de production de l'article défini pluriel au cas absolutif -ak.}

En basque, l'article défini pluriel (-ak 'les') au cas absolutif $(-\Theta)$ présente la même forme morphologique que l'article défini singulier (-a 'le/la') au cas ergatif (-k). Si l'on prend l'exemple du mot gizon 'homme', Gizonak peut renvoyer à un nom pluriel au cas absolutif (4) ou à un nom singulier au cas ergatif (5) comme cela est indiqué ci-après:

(4)

\author{
Gizon-ak- $\Theta$ \\ homme-art.pl-ABS \\ 'Les hommes'
}

\author{
Gizon-a-K \\ homme-art.sg-ERG \\ 'L'homme'
}

Lobjectif de ce test est donc de vérifier la production du déterminant pluriel au cas absolutif (-ak 'les') de manière à écarter un problème d'origine phonologique qui pourrait être lié à la position finale de l'occlusive / k/. Nous voulons avant tout nous assurer que l'enfant est capable de produire correctement ce phonème en fin de syntagme nominal. Le test en question est conçu à partir de dix verbes (ou locutions verbales) intransitifs qui sont : joan 'aller', sartu 'entrer', ateratu 'sortir', eskapatu 's'échapper', triste izan 'être triste', kexu izan 'être fâché(e)', kontent izan 'être content(e)', beldur izan 'avoir peur', lo izan 'être endormi(e)', iratzarri 'se réveiller', jostatu 's'amuser' et mintzatu 'parler'. Tous ces verbes requièrent la présence d'un sujet au cas absolutif dans la morphologie nominale.

L'épreuve, qui se déroule sous la forme d'un jeu, et consiste à donner la phrase au singulier (6) à partir d'une image en couleur présentant un seul personnage (animal ou humain): 
(6)

$$
\begin{aligned}
& \text { Sagua } \\
& \text { souris-art.sg-ABS }
\end{aligned}
$$

'La souris s'échappe'

$$
\begin{array}{ll}
\text { eskapatzen } & d a \\
\text { s'échapper-imperf. } & \text { aux.être }
\end{array}
$$

Puis une variante de cette image est présentée : elle comporte plusieurs personnages tandis que l'action reste inchangée. L'enfant est invité à reproduire la phrase qu'il a entendue précédemment au singulier cette fois-ci au pluriel (7), la transformation entraînant l'accord de l'auxiliaire izan 'être' à la $3^{e}$ personne du pluriel (dira 'sont') et au niveau nominal, l'ajout du déterminant pluriel ( $-a k$ 'les') :

$$
\begin{aligned}
& \text { Saguak } \\
& \text { souris-art.pl-ABS }
\end{aligned}
$$

'Les souris s'échappent'

$$
\begin{array}{ll}
\text { eskapatzen } & \text { dira } \\
\text { s'échapper-imperf. } & \text { aux.être }
\end{array}
$$

Parmi les réponses attendues, il se peut que l'enfant utilise la locution verbale intransitive ari 'en train de' mais cela ne gênera en rien la production. La phrase Saguak eskapatzen dira 'Les souris s'échappent' ou la suivante, Saguak eskapatzen ari dira 'Les souris sont en train de s'échapper' sont équivalentes dans le sens où l'enfant produit, dans les deux cas, le morphème -ak (marque du pluriel) au niveau nominal.

\subsubsection{Epreuve de production du déterminant singulier $-\mathrm{a}$ adjoint du cas ergatif $-\mathrm{k}$} en basque

Cette épreuve vise à faire produire des phrases avec un sujet porteur du cas ergatif en présence de verbes transitifs. Plus précisément, la structure des phrases attendues est la suivante : un sujet "agent» singulier marqué au cas ergatif $(-a-K)$ et un objet «patient » singulier marqué au cas absolutif $(-a-\Theta)$ ou un objet « patient » pluriel marqué au cas absolutif $(-a k-\Theta)$. Dans cette optique, le test est construit sur la base de cinq verbes transitifs : jan 'manger', ekarri 'apporter', hartu 'prendre', harrapatu 'attraper', pusatu 'pousser'. Chacun de ces verbes sert à l'élaboration de deux phrases distinctes, la différence se situant au niveau de l'objet qui varie en nombre. Concrètement, les images alternent entre des objets singuliers et pluriels. En conséquence, la production de l'enfant devrait respecter le nombre à travers l'emploi soit du déterminant singulier au cas absolutif -a 'le, la, l': oparia 'le cadeau'(8), soit du déterminant pluriel au cas absolutif -ak 'les': opariak 'les cadeaux'. Dans ce dernier cas, il faudra en plus que l'enfant accorde le verbe en insérant l'infixe -it- dans la forme verbale comme l'illustre l'exemple (9) ci-après :

$$
\begin{array}{lll}
\text { Neskak } & \text { oparia } & \text { ekartzen } \\
\text { fille-art.sg-ERG } & \text { cadeau-art.sg- } & \text { apporter- } \\
& \text { ABS } & \text { imperf. }
\end{array}
$$

'La fille apporte le cadeau'

ekartzen ditu

(9) apporter-imperf. $d u$ aux.avoir : accord sujet 3sg-objet 3sg aux.avoir : accord sujet 3sg-objet 3pl

'La fille apporte les cadeaux' 
S'agissant du matériel, les dix images conçues spécialement pour cette épreuve, mettent en scène des personnages humains (mutiko 'garçon', neska 'fille') ou des animaux (sagu 'souris', gatu 'chat') exerçant une action soit sur un autre être animé soit sur une entité inanimée (harri 'pierre', untzi 'boîte', goxoki/oñoñ 'bonbon', lore/lili 'fleur', liburu 'livre', haixtur 'ciseau(x)').

Le test est présenté de manière ludique avec l'intervention d'une poupée. Nous signalons à l'enfant que la poupée ne parle pas bien le basque, qu'elle ne connaît que quelques mots. Puis nous soumettons à l'enfant une première image suivie des mots soi-disant prononcés par la poupée. A partir des noms mutiko 'garçon', baloin 'ballon' (toujours indéterminés) et du verbe à l'infinitif botatu 'jeter', nous formulons la phrase-cible: Mutikoak baloina botatzen du 'Le garçon jette le ballon' dans laquelle le sujet mutiko 'garçon' prend le suffixe ergatif en tant qu'agent alors que l'objet baloin 'ballon' porte le cas absolutif.

L'ordre d'énonciation des mots étant toujours le même -Sujet (agent) Objet (patient) Verbe (action)-, il est possible que cela puisse influencer l'enfant dans sa production. C'est pourquoi les quatre dernières scènes de ce test sont destinées à la production de phrases réversibles. Elles présentent des situations où les personnages qui mènent l'action sont inversés : ce qui devrait obliger l'enfant à changer l'ordre d'apparition des participants ou à marquer différemment les personnages d'une image à l'autre. Le personnage « agent » (sujet) dans la première image devient « patient » (objet) dans la seconde induisant une modification des suffixes casuels.

\subsubsection{Epreuve de compréhension des cas absolutif et ergatif en basque}

Lobjectif est de voir si l'enfant fait la distinction entre l'article pluriel au cas absolutif $-a k$ et l'article singulier au cas ergatif $-a-k$ en désignant l'image correspondante à la phrase entendue. Pour cette épreuve, la plupart des dessins créés pour la production de l'article défini pluriel au cas absolutif et celle du cas ergatif ont été réutilisés pour formuler les phrasesstimuli tout en respectant les contraintes suivantes : 1) En premier lieu, il s'agit de proposer des situations admettant l'usage d'un verbe intransitif ou bien celui d'un verbe transitif pour décrire une même scène. Les verbes sélectionnés sont proches sur le plan sémantique mais relèvent d'une construction différente puisque les uns, c'est-à-dire les verbes intransitifs, nécessitent l'emploi du cas absolutif (ex. mintzatu 'parler') tandis que les autres, c'est-à-dire les verbes transitifs, requièrent le cas ergatif (ex. hitz egin 'parler') ; 2) Ensuite, nous avons joué sur la variation en nombre avec l'alternance de syntagmes nominaux sujets au singulier (se terminant par - $a$ à l'absolutif ou - $a k$ à l'ergatif) et de syntagmes nominaux sujets au pluriel (se finissant par -ak à l'absolutif) ; 3) Enfin, nous avons utilisé deux autres procédés qui sont la réversibilité des phrases et la dislocation du sujet à droite, c'est-à-dire le détachement du sujet après le verbe. Dans le cas des phrases réversibles, le sujet « agent» devient « patient » et inversement, le « patient » devient « agent ». A titre d'exemple, considérons le verbe transitif harrapatu 'attraper' et les deux arguments sagu 'souris' et gatu 'chat'. Il est alors possible de construire deux types de phrases respectant l'inversion des rôles, (10) Gatuak sagua harrapatzen du et (11) Saguak gatua harrapatzen du: 
(10)

Gatuak
chat-art.sg-ERG sagua

souris-art.sg-ABS harrapatzen

attraper-imperf. $d u$

aux avoir

'Le chat attrape la souris'

(11)

$\begin{array}{ll}\text { Saguak } & \text { gatua } \\ \text { souris-art.sg-ERG } & \text { chat-art.sg-ABS }\end{array}$

harrapatzen

attraper-imperf. $d u$

aux.avoir

'La souris attrape le chat'

Quant aux phrases construites selon le modèle de la dislocation du sujet à droite, celles-ci suivent l'ordre des mots OVS avec un sujet « agent » placé en dernière position comme dans la phrase (12) Gatua harrapatzen du saguak :

$\begin{array}{llll}\text { Gatua } & \text { harrapatzen } & d u & \text { saguak } \\ \text { chat-art.sg-ABS } & \text { attraper-imperf. } & \text { aux.avoir } & \text { souris-art.sg-ERG }\end{array}$

'(Elle) attrape le chat la souris'

Ce procédé permet de rompre avec l'ordre des mots canonique en basque SVO ou SOV suivant lequel le sujet « agent » serait positionné en premier.

\section{Résultats}

4.1. Production du déterminant défini pluriel au cas absolutif en basque

Lobjectif de ce test est de faire produire le morphème -ak représentant l'article défini pluriel marqué à l'absolutif).

\begin{tabular}{|cccc}
\multicolumn{1}{c}{ Enfants } & & Âge & Résultats / Pluriel -ak \\
\hline Bilingues simultanés & $\mathbf{n}^{\circ} \mathbf{1}$ & 6 ans & $10 / 12$ \\
& $\mathbf{n}^{\circ} \mathbf{2}$ & 7 ans & $12 / 12$ \\
& $\mathbf{n}^{\circ} \mathbf{3}$ & 6 ans & $12 / 12$ \\
Bilingues successifs L1 basque & $\mathbf{n}^{\circ} \mathbf{4}$ & 7 ans & $12 / 12$ \\
L1 basque & $\mathbf{n}^{\circ} \mathbf{5}$ & 5.5 ans & $12 / 12$ \\
L2 basque & $\mathbf{n}^{\circ} \mathbf{6}$ & 7 ans & $12 / 12$ \\
\hline Monolingues basques & $\mathbf{n}^{\circ} \mathbf{7}$ & 5.5 ans & $12 / 12$ \\
& $\mathbf{n}^{\circ} \mathbf{8}$ & 7 ans & $12 / 12$ \\
& $\mathbf{n}^{\circ} \mathbf{9}$ & 5 ans & $0 / 12$
\end{tabular}

Tableau 1. Epreuve de production du déterminant pluriel au cas absolutif en basque 
Globalement, la plupart des enfants bilingues et monolingues produisent l'article défini pluriel -ak 'les' sans difficultés dans la tâche guidée. Pourtant, quelques erreurs (ou réponses non attendues) peuvent surgir suivant les enfants. Cette question concerne plus exactement les bilingues précoces : simultané $\mathrm{n}^{\circ} 1$ ainsi que l'enfant monolingue bascophone $\mathrm{n}^{\circ} 9$. Le descriptif des erreurs est détaillé dans les lignes suivantes.

L'enfant bilingue simultané $\mathrm{n}^{\circ} 1$ produit deux réponses qui ne correspondent pas à la cible. Dans le premier cas, elle opte pour le déterminant numéral hiru 'trois' qui, selon la règle, implique l'usage d'un nom indéfini: hiru lapin 'trois lapins' au lieu du déterminant défini pluriel -ak 'les'. Même si l'enfant ne produit pas la forme attendue, c'est-à-dire lapinak 'les lapins', la réponse est néanmoins correcte. Dans le second cas, en revanche, elle associe le déterminant numéral bi 'deux' et l'article défini singulier - a 'le/la' comme dans l'exemple suivant : bi xolia (xoria)* 'deux l'oiseau*' avec substitution de la vibrante faible $\mathbb{\bigotimes}$ par la liquide //. Ici, par contre, il s'agit d'une erreur.

Quant à l'enfant monolingue $\mathbf{n}^{\circ} 9$, il ne suit pas la consigne : soit il répète la phrase au singulier telle qu'il l'entend sans aucune transformation (13); soit il produit le verbe intransitif au pluriel mais il omet le sujet (pluriel) au cas absolutif (14).

$\begin{array}{cll}\text { Stimuli } & \text { Réponses attendues } & \text { Réponses produites } \\ \text { (13) } \begin{array}{l}\text { neska xutitzen da } \\ \text { 'la fille se lève' }\end{array} & \begin{array}{l}\text { neskak xutitzen dira } \\ \text { 'les filles se lèvent' }\end{array} & \begin{array}{l}\text { neska xutitzen da } \\ \text { 'la fille se lève" }\end{array} \\ \text { (14) } \begin{array}{l}\text { neska jostatzen da } \\ \text { 'la fille joue' }\end{array} & \begin{array}{l}\text { neskak jostatzen dira } \\ \text { 'les filles jouent' }\end{array} & \begin{array}{l}\text { jostatzen dira } \\ \text { '(elles) jouent' }\end{array}\end{array}$

Ce comportement langagier explique le score nul obtenu (0/12). Toutefois, il convient de signaler que l'enfant maîtrise parfaitement le suffixe du pluriel comme nous avons pu nous en rendre compte durant l'entretien précédent la passation du test.

En résumé, malgré des erreurs minimes, les enfants testés sont aptes à produire le déterminant défini pluriel au cas absolutif $-a k$ dans des conditions de langage contrôlé. Ce premier résultat permet d'écarter un quelconque trouble phonologique quant à la production de la consonne sourde $/ \mathrm{k} /$. 


\begin{tabular}{|c|c|c|c|}
\hline \multicolumn{2}{|l|}{ Enfants } & Âge & Résultats / Ergatif -k \\
\hline \multirow[t]{3}{*}{ Bilingues simultanés } & $\mathrm{n}^{\circ} 1$ & 6 ans & $0 / 10$ \\
\hline & $\mathrm{n}^{\circ} 2$ & 7 ans & $1 / 10$ \\
\hline & $\mathrm{n}^{\circ} 3$ & 6 ans & $1 / 10$ \\
\hline Bilingues successifs Ll basque & $n^{\circ} 4$ & 7 ans & $9 / 10$ \\
\hline Ll basque & $n^{\circ} 5$ & 5.5 ans & $10 / 10$ \\
\hline L2 basque & $n^{\circ} 6$ & 7 ans & $7 / 10$ \\
\hline \multirow[t]{3}{*}{ Monolingues basques } & $\mathrm{n}^{\circ} 7$ & 5.5 ans & $8 / 10$ \\
\hline & $\mathrm{n}^{\circ} 8$ & 7 ans & $10 / 10$ \\
\hline & $\mathrm{n}^{\circ} 9$ & 5 ans & $9 / 10$ \\
\hline
\end{tabular}

Tableau 2. Epreuve de production du cas ergatif en basque

Comme le montre le Tableau 2, les monolingues bascophones $\mathbf{n}^{\circ} 7,8$ et 9 et les bilingues précoces successifs (L1 basque) $n^{\circ} 4$ et 5 et successif (Ll français) $n^{\circ} 6$ obtiennent de meilleurs résultats que les bilingues précoces simultanés $\mathbf{n}^{\circ} \mathbf{1}, \mathbf{2}$ et 3 . La dissociation au sein de la population bilingue précoce est donc visible ici : les enfants bilingues successifs ont un taux d'omissions du cas ergatif inférieur à trois (soit < 30\%) et se rapprochent des enfants contrôles monolingues tandis que les enfants bilingues simultanés effectuent de neuf à dix erreurs (soit entre $90 \%$ et $100 \%$ d'omissions du suffixe ergatif).

4.3. Compréhension des cas absolutif et ergatif en basque

L'enfant monolingue bascophone $\mathbf{n}^{\circ} \mathbf{9}$ n'a pas participé à l'épreuve de compréhension.

\begin{tabular}{|c|c|c|c|c|}
\hline \multicolumn{2}{|l|}{ Enfants } & Âge & $\begin{array}{c}\text { Résultats / } \\
\text { Compréhension }\end{array}$ & $\%$ \\
\hline \multirow[t]{3}{*}{ Bilingues simultanés } & $\mathrm{n}^{\circ} \mathbf{1}$ & 6 ans & $27 / 30$ & 90 \\
\hline & $\mathrm{n}^{\circ} 2$ & 7 ans & $29 / 30$ & 96.66 \\
\hline & $n^{\circ} 3$ & 6 ans & $28 / 30$ & 93.33 \\
\hline \multirow[t]{3}{*}{ Bilingues successifs Ll basque } & $n^{\circ} 4$ & 7 ans & $27 / 30$ & 90 \\
\hline & $n^{\circ} 5$ & $\begin{array}{l}5 \cdot 5 \\
\text { ans }\end{array}$ & (n’a pas participé) & - \\
\hline & $n^{\circ} 6$ & 7 ans & $28 / 30$ & 93.33 \\
\hline
\end{tabular}




$\begin{array}{lllccr}\text { Monolingue basque } & \mathbf{n}^{\circ} \mathbf{7} & \begin{array}{l}5.5 \\ \text { ans }\end{array} & 29 / 30 & 96.66 \\ & \mathbf{n}^{\circ} \mathbf{8} & 7 \text { ans } & 29 / 30 & 96.66 \\ & \mathbf{n}^{\circ} \mathbf{9} & 5 \text { ans } & \text { (n’a pas participé) } & -\end{array}$

Tableau 3. Epreuve de compréhension des cas absolutif et ergatif en basque

Globalement, les enfants bilingues et monolingues bascophones commettent très peu d'erreurs dans la compréhension des cas absolutif et ergatif en basque. Le taux de réussite s'échelonne entre 90\% et 97\% pour l'année 2010: ce qui représente au maximum $10 \%$ d'erreurs. Au plan quantitatif, tous les enfants ayant participé au test de compréhension des cas absolutif et ergatif réussissent cette épreuve en obtenant au minimum vingt-sept réponses correctes sur un total de trente stimuli.

D'un point de vue qualitatif, les erreurs commises par les enfants se classent en deux catégories majeures. On relève tout d'abord des confusions d'ordre morphémique entre le déterminant défini singulier au cas ergatif $-a-\mathrm{K}$ 'le/la' et le déterminant défini pluriel au cas

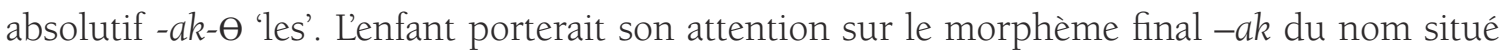
en première position tout en négligeant les indications véhiculées par l'auxiliaire qui signale la présence d'un sujet « agent » singulier et non pas d'un sujet pluriel. En second lieu, on observe des problèmes engendrés par la dislocation de sujet à droite qui viole l'organisation phrastique canonique SVO. Il semblerait que l'enfant considère le premier élément entendu comme étant le sujet " agent » sans prêter attention au marquage morphologique. Ainsi, une phrase du type (12) Gatua harrapatzen du saguak '(elle) attrape le chat, la souris' dans laquelle le sujet « agent » est disloqué à droite sera traitée comme une phrase du type Gatuak harrapatzen ditu saguak 'Le chat attrape les souris' qui suit l'ordre des mots SVO. L'enfant ne distingue donc pas les marques morphologiques et privilégie l'ordre des mots dans sa réponse².

En résumé, concernant l'ensemble des trois épreuves, nous pouvons dire que les enfants ont plus de difficultés dans la production du morphème marqué (ergatif) que celle du morphème non marqué (absolutif). En revanche, ils ont moins de problèmes sur le versant réceptif puisqu'on ne note pas d'erreurs importantes en compréhension.

\section{Discussion}

\subsection{Différences dans la maîtrise du cas ergatif (morphème marqué) selon les enfants}

Dans cette étude, les résultats montrent de la variabilité entre les enfants les classant en deux catégories: d'une part, les bilingues précoces successifs Ll basque qui gèrent mieux le morphème marqué -à la manière des monolingues bascophones- et d'autre part, les bilingues précoces simultanés qui éprouvent des difficultés. Lors d'un précédent article (Duguine,

2. Pour des exemples précis, nous renvoyons à notre thèse (Duguine, $2013: 188$ ). 
2014), nous avions tenté d'expliquer ce traitement variable du cas ergatif en mettant en avant l'existence de facteurs environnementaux comme la qualité et/ou la quantité de l'input parental. Un autre facteur serait à même de justifier ces différences : il s'agit du coût procédural causé par la production du cas ergatif. En allant plus loin, on peut se demander si ce déficit procédural est lié au type de bilinguisme. C'est-à-dire que, au vu de nos données, l'enfant qui apprend deux langues simultanément pourrait être désavantagé sur le plan des capacités implicites par rapport à celui qui n'a qu'une seule langue au départ (ici le basque), ce dernier bénéficierait pleinement de ce potentiel d'apprentissage et c'est pourquoi il maîtriserait plus aisément le marqueur de l'ergatif. Cela dit, il reste d'abord à prouver qu'il existe vraiment une différence entre les deux types de bilinguisme précoce, une dissociation qui est peut-être à nuancer de nos jours? Là s'ouvre un autre débat que nous n'aborderons pas dans le cadre de cet article.

\subsection{Ordre d'émergence des structures en fonction de leur degré de complexité}

Comme nous l'avons souligné plus haut, le degré de complexité structurale aurait un rôle dans la chronologie des acquisitions langagières puisque les formes simples sont acquises avant les formes complexes (Kail, 1997). Nos résultats montrent que le cas absolutif serait acquis avant le cas ergatif pour ce qui est de la morphologie nominale. L'hypothèse posée dans le cadre de cet article se vérifie donc ici : il semblerait que les enfants maîtrisent en priorité le morphème zéro puis le morphème marqué, probablement plus difficile à gérer. Dans le prolongement de cette idée, on peut se demander si la complexité structurale se définit en termes de nombre d'opérations à effectuer (autrement dit « quantitativement ») ou bien si elle est liée au type d'opération (soit « qualitativement »). Rappelons que, d'après Dordain et al. (1988) et Nespoulous (2006), la gestion de calculs plus profonds (de type syntaxique) serait plus difficile que celle des calculs superficiels (de type morphologique). Lors du traitement du message linguistique, la morphologie, plus superficielle, serait donc négligée par rapport à la syntaxe qui représenterait un niveau de traitement plus élevé. On peut donc en conclure l'existence probable d'une « hiérarchie » des calculs en fonction de leur degré de complexité. Il se peut que l'opération d'accord morphosyntaxique mobilise davantage l'attention et la mémoire de travail chez l'enfant que l'opération d'ajout de marques morphologiques et comme ses ressources cognitives sont (encore) limitées, l'enfant effectuerait des erreurs en morphologie. Transposé au cas du basque, ceci expliquerait les omissions du cas ergatif en production dans le sens où la construction d'une phrase transitive avec un sujet porteur du suffixe ergatif suppose un certain nombre d'opérations mentales et en particulier d'accords morphosyntaxiques entre le sujet, l'objet et l'auxiliaire en nombre et en personne.

\subsection{Existence probable de stratégies pour réduire le coût cognitif}

Suivant l'hypothèse des stratégies palliatives évoquée plus haut, le locuteur, lorsqu'il est en situation de difficultés langagières, peut recourir à des procédés linguistiques qui lui permettent malgré tout d'assurer une communication de l'information intelligible. Dans cette étude, nous observons que si les enfants bilingues ne produisent pas naturellement le marqueur ergatif, ils utilisent néanmoins d'autres procédés linguistiques pour pallier ce déficit, l'objectif étant, selon nous, de réduire le coût cognitif engendré par la production d'un tel 
marqueur. Dans l'épreuve de langage contrôlé, il semblerait que l'ordre des mots SVO soit une stratégie possible pour diminuer cette charge procédurale.

Rappelons que l'ordre des mots canonique en basque est SOV et que le marquage morphologique est nécessaire pour identifier le sujet du verbe transitif. Or, les enfants bilingues qui omettent le cas ergatif produisent généralement le schéma $S * V O$ suivant l'ordre « agentaction-patient ». Ainsi, l'information d'agentivité serait maintenue de par la place du sujet en première position, qui indique clairement son rôle et reléguant ainsi la nécessité du marquage du sujet au second plan. Parallèlement à cela, la question se pose de savoir si l'influence de l'autre langue (ici le français) existe. En effet, le français possédant un ordre des mots fixe de type SVO, il se peut que cela ait un impact sur la production phrastique en basque. En résumé, doit-on privilégier la piste de la dominance du français sur le basque qui expliquerait le schéma SVO (avec omission de l'ergatif) ? Celle d'un mécanisme de renforcement puisque les deux langues admettent la structure SVO ? Ou véritablement d'une stratégie syntaxique du sujet-agent en première position? Le débat reste ouvert.

\section{Conclusion}

Nous avons mis en évidence que : 1) les enfants n'avaient pas de problème de compréhension quant aux deux types de morphèmes (marqué et non marqué) ; 2) ils n'ont pas de problème phonologique dans la production du morphème /ak/ (test pluriel) et semblent maîtriser le cas absolutif sans difficultés ; 3) en revanche, les enfants bilingues ont plus de difficultés à gérer le morphème marqué (ergatif) par rapport aux monolingues bascophones : on constate en effet des différences dans le rythme d'acquisition ; 4) enfin, les enfants bilingues adoptent des stratégies linguistiques qui permettent d'alléger le coût cognitif engendré par la production de l'ergatif. Plus précisément, lorsque l'enfant omet le cas ergatif (ou le substitue par le morphème zéro), il peut employer l'ordre des mots canonique Sujet*-Verbe-Objet. Linformation indiquant l'agent de l'action est ainsi préservée grâce à la stratégie du sujet en première position. Ce procédé linguistique est observable chez la plupart des enfants bilingues. Ceci montre la capacité de ces enfants à s'adapter en situation de difficultés langagières et illustre par là même le potentiel cognitif de l'enfant dans le déploiement de stratégies de recours. On peut penser que l'environnement bilingue précoce solliciterait davantage les enfants bilingues dans la résolution de problèmes ainsi que dans le déploiement de stratégies par rapport aux enfants exposés à une seule langue. Ce qui aurait pour effet d'accroître certaines capacités chez les bilingues précoces alors qu'elles resteraient inusitées chez les monolingues. Ces stratégies pourraient bien révéler une forme d'adaptation de l'esprit humain qui doit prendre en compte à la fois les contraintes linguistiques et cognitives.

Par ailleurs, les résultats de notre étude ont montré un écart au sein de la population bilingue elle-même entre les bilingues précoces successifs ( $\mathrm{Ll}$ basque) qui produisent le cas ergatif comparativement aux bilingues précoces simultanés et successifs (L2 basque) qui ne le produisent pas. Au regard de ces résultats, nous pouvons en conclure que la gestion des morphèmes non marqués est plus facile que celle des morphèmes marqués. La question de l'impact sur l'ordre d'acquisition des structures en raison de leur coût procédural se pose pour les bilingues simultanés par rapport aux monolingues basques et aux bilingues successifs L1 basque. En effet, on peut se demander si l'organisation cérébrale entre les types de population est différente. Doit-on envisager l'existence d'un développement langagier de nature 
partiellement différent entre d'une part les enfants exposés à une langue, monolingues et bilingues précoces successifs (plus précisément ceux dont la langue maternelle serait identique à celle des monolingues) et d'autre part les enfants bilingues exposés à deux langues depuis la naissance (précoces simultanés) ? C'est une question qui mériterait d'être éclairée à la lumière d'autres recherches plus approfondies sur le bilinguisme précoce.

\section{Références}

Allières, Jacques. 1977. Les Basques. Paris : Presses Universitaires de France.

Allières, Jacques. 1979. Manuel pratique de basque. Paris : Picard.

Barreña, Andoni. 1995. Gramatikaren jabekuntza-garapena eta haur euskaldunak.Vitoria-Gasteiz : Université du Pays Basque.

Chanquoy, Lucile, Tricot, André \& Sweller, John. 2007. La charge cognitive. Théorie et applications. Paris: Armand Colin.

Gadet, Françoise. 1994. La genèse du concept de marque (1926-1931), Cahiers de l'ILSL 5. 81-92.

Garcia Iñaki, Barreña Andoni, Almgren Margareta, Arratibel Nekane, Barnes Julia \& Ezeizabarrena Maria José. 2011. MacArthur-Bates Communicatives Development Inventories tresnaren bertsio laburren egokitzapena euskarara : Komunikazio Garapenan Neurtzeko Zerrendaren bertsio laburrak. Uztaro 79. 49-73.

Ezeizabarrena, Maria José \& Larranaga, Maria Pilar. 1996. Ergativity in Basque: a problem for language acquisition? Linguistics 34. 955-991.

Ezeizabarrena, Maria José. 2012. The (in)consistent ergative marking in early basque : Ll vs. child L2. Lingua. 303-317.

Huarte, Maider. 2007. Ergatiboaren jabekuntza : ikerketa esperimental bat eta beraren emaitzak. Thèse de doctorat. Vitoria Gasteiz : Université du Pays Basque.

Huarte, Maider 2007. The acquisition of Basque Ergative case. An experimental study, Anuario del Seminario de Filología vasca "Julio de Urquijo" XLI(2). 131-144.

Jakobson, Roman \& Waugh, Linda. 1980. La charpente phonique du langage. Paris : Editions de Minuit.

Jakobson, Roman. 1969. Langage enfantin et aphasie. Paris : Editions de Minuit.

Kail, Michèle. 1997. Variations linguistiques et contraintes cognitives dans le traitement et le développement du langage. In Catherine Fuchs \& Stéphane Robert, Diversité des langues et représentations cognitives 210-226. Paris: Ophrys.

Kolk, Herman. 2001. Does agrammatic speech constitute a regression to child language? A threeway comparison between agrammatic, child and normal ellipsis. Brain and Language 77. 340-350.

Delage, Hélène. 2008. Evolution de l'hétérogénéité linguistique chez les enfants sourds moyens et légers: étude de la complexité morphosyntaxique. Thèse de doctorat. Tours: Université François-Rabelais.

Delage, Hélène et Frauenfelder, Ulrich Hans. 2012. Développement de la mémoire de travail et traitement des phrases complexes: quelle relation? Actes du CMLF 2012. 3e Congrès Mondial de Linguistique Française.

Dordain, Monique, Nespoulous, Jean-Luc \& Louboff, Catherine. 1988. De l'agrammatisme 
à la dyssyntaxie : étude longitudinale d'un cas d'agrammatisme. Folia Phoniatrica 40. 277-283.

Dordain, Monique et Nespoulous, Jean-Luc. 1992. Le traitement des syntagmes nominaux par l'aphasique agrammatique: une illustration des corrélations (directes ?) existant entre « complexité structurale » et « complexité procédurale ». Revue de Neuropsychologie 2(4). 395-417.

Duguine, Isabelle. 2013. Le développement du langage oral chez l'enfant bilingue basque-français en contexte d'acquisition simultanée vs. successive des langues. Etude de la maîtrise du cas ergatif en basque et du genre grammatical en français. Thèse de doctorat. Toulouse : Université Toulouse 2-Le Mirail.

Duguine, Isabelle, Köpke, Barbara \& Nespoulous, Jean-Luc. 2014. Variations dans l'acquisition du marqueur ergatif en basque par des enfants bilingues basques-français. Language Interaction and Acquisition 5(2). 227-251.

Duvignau, Karine, Gaume, Bruno \& Nespoulous, Jean-Luc. 2004. Proximité sémantique et stratégies palliatives chez le jeune enfant et l'aphasique. Revue Parole $\mathrm{n}^{\circ}$ spécial. In JeanLuc Nespoulous \& Jacques Virbel (ed.), Handicap langagier et recherches cognitives : apports mutuels 31-32. 219-255. Mons : Université de Mons-Hainaut.

Nespoulous, Jean-Luc. 1996. Les stratégies palliatives dans l'aphasie. Rééducation Orthophonique 34(188). 423-433.

Nespoulous, Jean-Luc. \& Moreau, Nadine. 1998. Repair strategies and the production of segmental errors in aphasia : epentheses vs. syncopes in consonantal clusters. In Evy Visch-Brink \& Roelien Bastiaanse (eds.), Linguistic levels in aphasia, 133-145. San Diego: Singular publishing group, inc.

Nespoulous, Jean-Luc \& Virbel, Jacques. 2004. Apport de l'étude des handicaps langagiers à la connaissance du langage humain. Revue Parole 29-30. 5-42.

Nespoulous, Jean-Luc. 2004. Linguistique, pathologie du langage et cognition : Des dysfonctionnements langagiers à la caractérisation de l'architecture fonctionnelle du langage. In Fuchs, Catherine. (Ed.), La linguistique cognitive. 171-194. Paris: Ophrys \& Editions de la MSH.

Nespoulous, Jean-Luc. 2006. La morphologie dans tous ses états : linguistique, psycholinguistique et neuropsycholinguistique. Une composante linguistique universelle ? De la labilité de sa gestion par le cerveau/esprit humain. Rééducation Orthophonique 225. 7-18.

Oyharçabal, Bernard. 2003. Le basque. In Bernard. Cerquiglini (ed.), Les langues de France, 5968. Paris : Presses Universitaires de France.

Oyharçabal, Bernard. 2011. La langue basque. In Emilio Bonvini, Joëlle Busuttil \& Alain Peyraube (ed.), Dictionnaire des langues, 857-869. Paris : Presses Universitaires de France.

Sahraoui, Halima. 2009. Contribution à l'étude des stratégies compensatoires dans l'agrammatisme. Approche neuropsycholinguistique de la performance de six locuteurs agrammatiques en production orale: caractérisation quantitative et fonctionnelle des variabilités. Thèse de doctorat. Toulouse : Université Toulouse II- Le Mirail.

Sahraoui, Halima \& Nespoulous, Jean-Luc. 2008. Déficit et conduites adaptatives dans la production verbale agrammatique : variabilités à propos d'un cas. Glossa 104. 42-56. 